\title{
O processo de hospitalização sob a ótica do paciente
}

\author{
Marcella Maria Pereira iD \\ Faculdade de Medicina de Marília - Marília (SP) - Brasil \\ Katia Terezinha Alves Rezende (D) \\ Faculdade de Medicina de Marília - Marília (SP) - Brasil \\ lone Ferreira Santos (iD \\ Faculdade de Medicina de Marília - Marília (SP) - Brasil
}

Silvia Franco Da Rocha Tonhon (iD

Faculdade de Medicina de Marília - Marília (SP) - Brasil

\section{RESUMO}

Objetivo: Analisar o processo de internação na perspectiva do paciente internado, o conhecimento dele sobre o (s) diagnóstico (s) que resultou (ram) na internação, sobre o plano terapêutico aplicado e sua percepção sobre o processo de internação. Métodos: Pesquisa qualitativa realizada em 2016 em uma unidade de internação do Hospital das Clínicas da Faculdade de Medicina do interior paulista com um total de 13 pacientes entrevistados. A coleta de dados foi realizada por meio de entrevista semiestruturada e empregada análise de conteúdo na modalidade temática. Resultados: Identificaram-se três categorias: conhecem o diagnóstico e o plano terapêutico, satisfação com o processo de internação; conhecem o diagnóstico e parcialmente o plano terapêutico, insatisfação com o processo de internação; conhece o diagnóstico e desconhece o plano terapêutico, consciência da necessidade de internação. Conclusão: Constatou-se que os pacientes internados têm conhecimento sobre o diagnóstico que motivou sua internação e o plano terapêutico submetido, e que essas orientações foram transmitidas pelo médico de maneira mecanizada, não sendo possível identificar uma relação dialógica entre o profissional e o paciente.

Descritores: Assistência Integral à Saúde; Pacientes Internados; Comunicação em Saúde.

\section{INTRODUÇÃO}

A experiência vivenciada na Unidade de Prática Profissional (UPP), unidade educacional do Curso de Enfermagem da Faculdade de Medicina de Marília (Famema) desenvolvida nos serviços de atenção terciária no $3^{\circ}$ ano da graduação, mostrou que alguns pacientes desconhecem seu diagnóstico clínico bem como a finalidade dos cuidados necessários para tanto. Sendo assim, surgiu a hipótese de que o plano de cuidados é desenvolvido pelos profissionais de saúde desconsiderando a autonomia da pessoa internada ${ }^{(1)}$.

Acreditou-se que isto pode ocorrer por fragilidades da equipe de saúde e dos pacientes; os médicos desvalorizarem a necessidade do paciente saber sobre seu diagnóstico clínico e seu plano terapêutico; e os enfermeiros e a equipe de enfermagem não possuem autonomia para essa orientação. Além disso, partiu-se do pressuposto de que os pacientes possuem escolaridade insuficiente para esta compreensão(1).

Em um estudo(2) para investigar a percepção que o paciente com leucemia atribui sobre o processo de internação hospitalar e as formas de enfrentamento da doença, identificou-se quatro categorias a partir do processo de internação, sendo elas: reconhecimento de sinais e sintomas da leucemia, sentimentos frente à doença, enfrentamento da doença e do processo de internação, mudanças nos hábitos de vida relacionados à internação e o tratamento.

Os sentimentos vivenciados pelos pacientes no adoecimento por acidente vascular encefálico (AVE) caracterizam-se por medo, tristeza, perplexidade diante do surgimento inesperado da doença e, por fim, alívio e desejo de mudança. Os autores perceberam nas falas, inicialmente, o sentimento de medo da morte. Além desse sentimento, identificaram

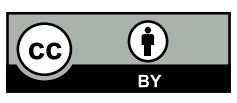

Este artigo está publicado em acesso aberto (Open Access) sob a licença Creative Commons, que permite uso, distribuição e reprodução em qualquer meio, sem restrições, desde que o trabalho seja corretamente citado. 
apreensão relacionada às sequelas decorrentes do AVE e, por sua vez, à incapacidade para as atividades laborais. A partir do momento em que os indivíduos admitiam a patologia como circunstância real em suas vidas, passaram a buscar explicações para o evento e tentaram reconhecer as condições causais que levaram ao surgimento da doença. É apontado pelos autores que os pacientes elaboraram suas próprias justificativas, enquanto outros se apropriaram de informações fragmentadas emitidas pelos profissionais de saúde para construir suas explanações relacionadas ao $\mathrm{AVE}^{(3,1)}$.

Outro estudo(4) traz necessidades vivenciadas por pacientes no pós-operatório de cirurgia cardíaca, os quais referem que para amenizar os sentimentos relacionados a essa experiência, deve-se prestar um cuidado de enfermagem que supre as necessidades dos indivíduos.

Em uma pesquisa ${ }^{(5)}$ realizada em um Hospital Universitário de Londrina no setor de isolamento, percebeu-se, na fala dos entrevistados, que as orientações fornecidas foram superficiais, informando apenas que eles possuíam uma bactéria. Os autores destacaram que, assim como a equipe de saúde, o paciente, quando informado e esclarecido, pode ser um participante ativo no seu autocuidado e na prevenção da disseminação da Infecção Hospitalar (IH).

Em uma Unidade de Terapia Intensiva (UTI) identificam-se quatro aspectos que precisam ser considerados para contribuir com a melhoria na qualidade do cuidado e favorecer a adaptação em um ambiente tão estigmatizado. São eles: referentes à sua visão de UTI anterior à internação, apontando esta unidade como um ambiente relacionado com a morte; ao cliente internado na UTI possuir uma concepção positiva do ambiente e da equipe de trabalho em comparação à enfermaria; a UTI como um ambiente que oferece serviço especializado e equipamentos tecnológicos avançados e de alta precisão que são essenciais para recuperação; ao estresse gerado por essa unidade relacionado aos fatores como luz acesa, interrupção do sono pela equipe, monitorização contínua, ficar parado sem nada para fazer e falta de privacidade para as necessidades fisiológicas como agentes estressores ${ }^{(6,1)}$.

Diante da problemática e das hipóteses mencionadas acima, surgiram inquietações que emergiram as seguintes questões: Qual é o conhecimento dos pacientes internados sobre o (s) seu (s) diagnóstico (s) que resultou na internação bem como sobre o plano terapêutico definido pela equipe de saúde? Quem é / são responsável (is) por essa informação? Qual é a percepção destes pacientes sobre o processo de internação?

Assim o objetivo deste estudo foi analisar o processo de internação na perspectiva do paciente internado, o conhecimento dele sobre o (s) diagnostico (s) que resultou (ram) na internação, sobre o plano terapêutico aplicado e sua percepção sobre o processo de internação. Identificar quem é/são o (s) profissional (is) responsável (is) pela informação sobre o motivo da internação e plano terapêutico.

\section{MÉTODOS}

A fim de alcançar os objetivos apresentados foi realizada esta pesquisa qualitativa(7) realizada no Hospital das Clinicas - Unidade I (HC-I) do complexo Famema. A faculdade é uma autarquia do Governo do Estado de São Paulo, ligada à Secretaria de Desenvolvimento Econômico, Ciência e Tecnologia do Estado de São Paulo, portanto pública, oferecendo os cursos de Medicina e Enfermagem ${ }^{(8)}$.

$\mathrm{O} \mathrm{HC}$ I é unidade de referência para 62 municípios da região, ofertando atenção especializada nos diferentes níveis de complexidade, operacionalizando 111 leitos, sendo 24 distribuídos em duas Unidades de Terapia Intensiva ${ }^{(8)}$. $O$ estudo foi realizado em uma das seis unidades de internação. Os pacientes internados são predominantemente da faixa etária de 60 a 70 anos, representando $43 \%$ dos pacientes desta unidade.

Foram entrevistados todos os internados no período de 18 a 22 de janeiro de 2016, independentemente da idade, sexo, morbidade e tempo de internação. Os impossibilitados de realizar a entrevista, ou seja, que apresentaram dificuldade de se comunicar oralmente e os com alteração do estado mental foram excluídos da pesquisa.

Realizou-se a coleta de dados por meio da entrevista semi-estruturada gravada e transcrita, beira leito, utilizando um instrumento composto por duas partes. Uma constava de dados de identificação: nome, data de nascimento, sexo, escolaridade, estado civil, ocupação; a segunda parte constava das questões norteadoras: Qual o motivo de sua internação? Qual é (são) o (s) seu (s) problema (s) de saúde que levaram a internação? De que maneira você/ senhor (a) obteve este conhecimento? Qual é o plano terapêutico indicado para você/senhor (a)? De que maneira você/senhor (a) obteve este conhecimento? Como tem sido o seu processo de internação? Como se sente com a internação, desde a admissão até hoje?

Dentre os pacientes internados, três se encontravam em precaução de contato por diferentes tipos de infecção, três traqueostomizados e um com sequelas de um AVE, os quais foram excluídos. Assim, no primeiro dia de coleta, estavam disponíveis sete pacientes, no segundo dia não houve admissão, no terceiro dia houve uma admissão, no 
quarto dia houve quatro admissões, no quinto e último dia havia uma admissão, totalizando, portanto, 13 entrevistas.

Os dados foram submetidos à análise de conteúdo na modalidade temática ${ }^{(7)}$. A análise temática consiste em descobrir os núcleos do sentido que compõem uma comunicação, cuja presença ou frequência signifiquem algo para o objeto analítico visado. Essa modalidade compreende três etapas: Pré-Análise que abrange a escolha dos documentos a serem analisados e a retomada das hipóteses e dos objetivos iniciais da pesquisa; Exploração do material, operação classificatória que visa alcançar o núcleo de compreensão do texto, busca encontrar categorias que são expressões ou palavras significativas em função das quais o conteúdo de uma fala será organizado; Tratamento dos resultados obtidos e Interpretação, são submetidos a operações estatísticas simples ou complexas que permitem colocar em relevo as informações obtidas, a partir daí propõe inferências e realiza interpretações ${ }^{(7)}$.

Da análise dos dados obtidos por meio das entrevistas emergiram três categorias analíticas: A) Conhecem o diagnóstico e o plano terapêutico, satisfação com o processo de internação; B) Conhecem o diagnóstico e parcialmente o plano terapêutico, insatisfação com o processo de internação; C) Conhece o diagnóstico e desconhece o plano terapêutico, consciência da necessidade de internação.

O estudo foi aprovado pelo Comitê de Ética em Pesquisa da Faculdade de Medicina de Marília, sob o Parecer $n^{0}$ 1.312.201. Os pacientes foram incluídos no estudo após leitura e assinatura do Termo de Consentimento Livre e Esclarecido (TCLE).

\section{RESULTADOS E DISCUSSÃO}

\section{Caracterização dos entrevistados}

Dos 13 pacientes entrevistados, oito (62\%) pertencem a faixa etária acima de 60 anos, três $(23 \%)$ entre 20 a 39 anos e dois (15\%) de 40 a 59 anos. Em relação ao sexo, oito (62\%) pacientes eram do sexo feminino. Quanto à escolaridade, cinco (39\%) tinham o ensino fundamental incompleto ou completo, dois (15\%) eram analfabetos, dois $(15 \%)$ possuíam ensino superior, um $(8 \%)$ não frequentou a escola, porém sabia ler e escrever, dois ( $8 \%)$ com ensino médio incompleto ou completo e uma (8\%) entrevistada não soube referir. Referente ao estado civil dos pacientes, havia cinco $(39 \%)$ casados, três $(23 \%)$ solteiros, três $(23 \%)$ viúvos e dois $(15 \%)$ separados.

Constatou-se que a maioria dos pacientes era composta por idosos e não possuíam o ensino fundamental completo, condições que podem dificultar a construção do conhecimento a respeito do diagnostico que resultou na internação bem como a finalidade dos cuidados necessários para tanto.

\section{A - Conhecem o diagnóstico e o plano terapêutico, satisfação com o processo de internação}

Em geral, os entrevistados conhecem o diagnóstico e o seu plano terapêutico. Estão satisfeitos com a internação, pois estão recebendo os cuidados necessários. Adquiriram esse conhecimento por intermédio do médico:

"[...] é a válvula mitral que deu problema, tenho duas lesões na válvula mitral e hipertensão pulmonar também [...] a médica examinou [...] estão me dando remédio, para afinar o sangue, [...], está dando de tudo [...] agora eles estão me pondo na cadeira aqui e me levando no banheiro para tomar banho, mas tem que ser um banho muito rápido e já voltar para cá [...]." (P3)

A autonomia do paciente pode ser proporcionada por meio da clínica ampliada realizada pela equipe de saúde, já que a mesma surge como possibilidade de uma mudança do modelo tradicional do processo de trabalho, transformando o sujeito inerte, que deve cumprir o que se define como cuidado, em sujeito ativo, podendo contribuir para o planejamento e desenvolvimento deste ${ }^{(9)}$.

A clínica ampliada pressupõe o estabelecimento de vínculo, dando voz ao paciente, escutando-o, não só para tratar o problema de saúde, mas para uma relação dialógica, que além de informações e esclarecimentos de dúvidas, seja um acolhimento de preocupações e medos ${ }^{(10)}$.

A construção do vínculo profissional-paciente é estabelecida em forma de comunicação. O valor da comunicação é claro e indispensável, visto que uma simples palavra bem colocada poderá criar um vínculo de confiança. Ao estabelecer esse vínculo, o profissional pode escutar com ética as necessidades do paciente, criando um laço de confiança, consequentemente, o bem-estar. A comunicação demonstra ser uma maneira de estabelecer a relação de ajuda ao paciente no processo-doença ${ }^{(11)}$.

Identificou-se pelas entrevistas que as informações são diretas, não aparece um diálogo entre os profissionais e o paciente, pois apesar de conhecerem o diagnóstico e o plano terapêutico; suas preocupações, angústias e medos em relação a este processo deixaram de ser acolhidas pela equipe de saúde. Nesse contexto, o atendimento que 
prioriza a escuta propicia o vínculo paciente-profissional, o que pode humanizar a assistência. No presente estudo, identificaram-se a falta de informações por parte de alguns profissionais da saúde com relação ao paciente e uma comunicação ineficaz entre ambos os sujeitos.

Esse resultado diverge do encontrado em estudo realizado com usuários do SUS em um município do Rio de Janeiro, o qual identificou como satisfatórios elementos constituintes das relações entre os sujeitos, sendo esse um dos melhores itens avaliados. Ainda, na pesquisa, avaliou-se como efetiva a dimensão da informação e comunicação, com destaque positivo no que se refere à explicação do profissional sobre exames e sintomas da doença. Tais elementos corroboram para a humanização da assistência aos usuários, destacando o esclarecimento do profissional, acolhimento e escuta qualificada ${ }^{(12)}$.

Assim, um estudo ${ }^{(13)}$ esclarece que $o$ atendimento multiprofissional ao paciente submetido a procedimentos intervencionistas em cardiologia, de forma a esclarecer suas dúvidas e questionamentos acerca dos seus sintomas, com foco no indivíduo e não na doença, mostrou-se efetivo na redução do estresse emocional do paciente e no aumento da sua expectativa sobre o sucesso do tratamento.

Uma das entrevistadas dessa pesquisa se sente cansada e angustiada com o processo de internação visto que tem mais de um mês que está internada e ainda não houve a resolução do problema de saúde. Obteve o conhecimento pelo médico por um exame de imagem:

"[...] estou com uma hérnia de disco na coluna, na cervical [...] Vai fazer cirurgia [...] já fui duas vezes para sala de cirurgia para fazer, só que falta instrumento, então agora eu vou ser transferida [...] Olha já faz um mês e pouco que eu estou aqui, então está assim tudo bem, mas eu já estou cansada de ficar, porque enquanto eu sabia que ia ter que fazer cirurgia aqui você espera, agora que eu sei que vou ser transferida, então às vezes você começa a ficar meio angustiada sabe [...]." (P13)

Há necessidade de compartilhamento com os usuários dos diagnósticos e condutas em saúde, tanto individual quanto coletivamente ${ }^{(11)}$. A fala a seguir ilustra esse compartilhamento:

"[...] um sintoma muito ruim e tudo, agora descobriu um tumor na cabeça [...] procurei um médico clínico, aí o doutor pediu a ressonância, que descobriu [...] ele falou olhando dentro do meu olho [...] Para fazer a cirurgia [...] eu tomo os meus remédios mesmo para cabeça [...] Fácil não é, mas, eu tenho muito conhecimento [...]." (P5)

Quanto mais longo for o seguimento do tratamento maior a necessidade de participação e adesão do indivíduo ao projeto terapêutico e maior será o desafio para equipe de saúde e pessoa que necessita do cuidado ${ }^{(11)}$.

Outro entrevistado conhece o seu diagnóstico e plano terapêutico, referindo que o processo de internação foi satisfatório, pois recebeu medicações diante da sua dor. Obteve as informações pelo médico e este lhe proporcionou acesso ao serviço público:

"[...] Para operar do intestino, não, do estômago [...] Tem tudo, e está com infeç̧ão também [...] Fui ao médico [...] ele tentou tratar, mas não melhorou, aí ele falou, vai precisar internar aí eu falei, ó eu não tenho condição de pagar particular, ai ele mandou para cá [...] Está bom tem nada não [...] o tratamento é aqui, soro [...] Quando começa a doer, eles vem aqui e aplicam remédio pra tirar a dor [...]." (P6)

Ao repensar o modelo de gestão dos cuidados de Enfermagem em uma unidade de internação, na perspectiva da integralidade, considera-se a importância de resgatar as singularidades e complexidades dos usuários, os quais são seres humanos únicos e necessitam ser atendidos nas suas multidimensionalidades ${ }^{(14)}$. $O$ atendimento integral ou princípio da integralidade são termos que apresentam o mesmo significado e se constituem na atualidade um enorme desafio para o setor da saúde. Com a integralidade procura-se uma assistência qualificada, ampliada, transformadora, centrada na pessoa como um todo, respeitando os seus valores e direitos, não aceitando a redução da mesma à doença e ao aspecto biológico.

A integralidade das ações em saúde favorece a atuação responsável dos profissionais envolvidos com o cuidar/ tratar/curar. Constitui-se no alicerce para o alcance de uma melhor qualidade das ações e serviços voltados para a promoção da saúde, prevenção, recuperação e reabilitação(15).

O cuidado de forma idealizada, recebido/vivido pelo paciente é somatório de um grande número de pequenos cuidados parciais que vão se complementando, entre os vários cuidadores. Nesse sentido, uma complexa trama de atos, de procedimentos, de fluxos, de rotinas, de saberes, num processo dialético de complementação e disputa, conformará o cuidado em saúde. A integralidade depende da forma como se articulam as práticas dos trabalhadores do hospital ${ }^{(16)}$. 
Alguns pacientes que desconhecem o seu diagnóstico bem como o seu plano terapêutico são pessoas idosas com dificuldade de compreensão e para os seus cuidados, possuem uma dependência da família. Assim é que a família detém o conhecimento e este foi obtido pelo médico. Um deles não obteve as informações de nenhum profissional da saúde por opção da família devido ao prognóstico. Os entrevistados têm consciência que é necessária a internação para o tratamento. A fala a seguir mostra este núcleo de sentido:

“[...] Por causa do estômago [...] A comida não parava no estômago [...] Aí eu falei vou procurar o médico, foi que eu comecei a tratar [...] Eu não tenho sabedoria não, quem sabe são eles [...] Eu não sei ler, não entendo. Então quem sabe são só eles, eu não estou sabendo de nada. Eu sei que é dor no estômago [...] Bom não é, mas tem que ficar [...]." (P9)

\section{B - Conhecem o diagnóstico e parcialmente o plano terapêutico, insatisfação com o processo de internação}

Uma paciente possui conhecimento sobre o diagnóstico; em relação ao plano terapêutico menciona as medicações em uso e não refere o acompanhamento nutricional e psicológico. Não está satisfeita por estar internada, pois possui um filho para cuidar e a equipe de saúde não Ihe proporciona alta hospitalar. A entrevistada foi encaminhada para cirurgia sem saber o diagnóstico, que não estava definido. Após a intervenção cirúrgica o médico explicou o que havia acontecido:

“[...] tirei um ovário, uma trompa e o apêndice [...] teve que me abrir, porque não estava achando o problema [...]. Depois que eu operei, o médico falou [...] Antibiótico e soro [...] Horrível [...] Porque eu tenho um filho de quarenta dias, eu quero ir embora e eles não deixam [...]." (P2)

Outra entrevistada conhece seu diagnóstico e em relação ao plano terapêutico, menciona as medicações em uso, mas omite a oxigenoterapia. Além desse conhecimento, sabe que é portadora de uma infecção sexualmente transmissível há dez anos. Acredita que o processo de internação poderia ser melhor se fosse como ela queria, ou seja, não permanecer restrita ao leito, e traz a seguinte fala:

“[...] Pneumonia e outros probleminhas mais [...]. Eu sei que eu estou tomando seis tipos de antibióticos, agora pra que que é [...] eu gosto de ficar aqui, o ruim é que eles querem amarrar para não perder veia, e aí eu fico agoniada, é isso, mas se for para ficar aqui um mês eu fico, mas do meu jeito também, porque eu não vou ficar amarrada [...]." (P12)

Nas falas acima identifica-se uma valorização do enfoque biomédico em detrimento do social e psicológico, tal compreensão é fruto do trabalho em saúde hegemônico no Brasil, o qual foca em ações de recuperação e reabilitação da saúde física das pessoas.

Complementando a discussão sobre a comunicação entre equipe de saúde e pacientes/familiares, é possível inferir que alguns profissionais demonstraram uma forma inapropriada de se comunicar com os mesmos ${ }^{(17)}$. Dentre as muitas correntes teóricas que contribuem para o trabalho em saúde, a cartilha do Ministério da Saúde distinguiu três grandes enfoques para mostrar o porquê precisamos da clínica ampliada: o biomédico, o social e o psicológico ${ }^{(18)}$.

A Clínica Ampliada busca se constituir numa ferramenta de articulação e inclusão dos diferentes enfoques e disciplinas. A Clínica Ampliada reconhece que, em um dado momento e situação singular, pode existir uma predominância, uma escolha, ou a emergência de um enfoque ou de um tema, sem que isso signifique a negação de outros enfoques e possibilidades de ação(18).

O princípio da autonomia, denominação mais comum pela qual é conhecido o princípio do respeito às pessoas, exige que aceitem-se que elas se autogovernem, ou sejam autônomas, quer nas suas escolhas, quer nos seus atos $^{(19)}$. O reconhecimento da autonomia das pessoas, além de possibilitar a humanização, é questão central, tanto no âmbito moral e jurídico. O desconhecimento e o desrespeito dos direitos dos pacientes e a não legitimação de seu poder de escolha podem ser considerados como causas dos processos de cuidados desumanizados ${ }^{(20)}$.

No ambiente hospitalar, a capacidade decisória do ser humano pode encontrar-se cerceada por questões administrativas e burocráticas inerentes ao contexto institucional hospitalar. A fragilidade do ser humano acometido por alguma doença, e dependente de outro, potencializa ainda mais a dificuldade de tomar decisões autônomas e deposita nos provedores do cuidado hospitalar a responsabilidade de ver esse sujeito como pessoa com necessidades, valores, experiências e intenções próprias. A integração e a participação efetiva do ser humano hospitalizado em seu plano terapêutico revertem em mudanças sociais, estruturais e de relações de poder estabelecidas nas instituições de saúde a partir do momento da hospitalização(21). 


\section{C - Conhece o diagnóstico e desconhece o plano terapêutico, consciência da necessidade de internação}

Uma entrevistada possui conhecimento sobre o diagnóstico, porém desconhece o plano terapêutico. A entrevistada sabe da necessidade da internação para o tratamento. Adquiriu esse conhecimento por meio do médico:

“[...] Eu tive que cortar um pedaço da perna [...] Por causa de trombose [...] Estão cuidando muito bem [...] Não [...] Sei lá porque [...] Tem que cuidar. Tem hora que foge a idéia, foge um pouquinho, mas a gente sabe que é aqui mesmo, é aqui mesmo que tem que ficar [...]." (P1)

A ausência da comunicação, em suas diversas formas, durante o cuidado ao paciente, pode mostrar uma negligência na dimensão psicossocial do paciente, pois ele possui seus medos e anseios. A mecanização dos cuidados evidencia que muitos profissionais ainda se detêm apenas ao tratamento e cura do corpo doente ${ }^{(22)}$.

A proposta de interação e do diálogo é essencial para a construção da humanização, pois a capacidade de falar e ouvir perpassa as questões de empatia e valorização dos diferentes sujeitos. Pensar nas relações de empatia é se posicionar no lugar do outro, transformando as questões relativas ao cuidar, correlacionado, assim, a fatores da subjetividade dos mesmos ${ }^{(23)}$.

Acredita-se que haja uma vasta literatura sobre a humanização do cuidado, mas ainda está distante da realidade dos usuários e trabalhadores da saúde. Este contexto estimula à reflexão, quase constante, sobre a estruturação do sistema de cuidados e sobre os seus significados para esses seres humanos/pacientes. Existem outras formas/ maneiras de cuidar em nível hospitalar. Os profissionais da saúde que atuam na rede hospitalar enfrentam o desafio de repensar e de valorizar o princípio da autonomia das pessoas. Há um longo caminho a ser percorrido para, quem sabe, ressignificar o processo de cuidado, das relações, dos sentimentos e do respeito à dignidade humana nos hospitais ${ }^{(24)}$.

Os resultados da presente pesquisa demonstraram que o médico foi o protagonista de transmitir a informação aos pacientes entrevistados ou para seus familiares sobre seu diagnóstico clínico. O Código de Ética Médica mostra que é vedado a este profissional deixar de informar ao paciente o diagnóstico, o prognóstico, os riscos e os objetivos do tratamento, salvo quando a comunicação direta possa lhe provocar danos, devendo, nesse caso, a comunicação deve ser dirigida ao seu representante legal. Esta ação mencionada ficou restrita ao médico, prática esta que incomoda, pois a realidade do trabalho em saúde revela que se apresenta como demanda para o enfermeiro e este não a realiza. Espera-se que este profissional apresente competência para tanto e não o faz por não possuir autonomia. Retomando o código de ética médica, observa-se que não exista algum artigo que define que esta ação é exclusiva deste profissional e no código de ética de enfermagem não existe artigo que proíbe estes profissionais de exercer essa função. A fim de refletir sobre a autonomia do enfermeiro nesta ação, alguns artigos do código de ética apontam que devem prestar adequadas informações à pessoa, família e coletividade a respeito dos direitos, riscos, benefícios e intercorrências acerca da Assistência de Enfermagem; colaborar com a Equipe de Saúde no esclarecimento da pessoa, família e coletividade a respeito dos direitos, riscos, benefícios e intercorrências acerca de seu estado de saúde e tratamento; proteger a pessoa, família e coletividade contra danos decorrentes de imperícia, negligência ou imprudência por parte de qualquer membro da Equipe de Saúde; participar da prática profissional multi e interdisciplinar com responsabilidade, autonomia e liberdade. Ao refletir essa questão ética do ponto de vista da integralidade, compreende-se que o cuidado em saúde não se faz sozinho e que mesmo que um médico esteja sozinho, de portas fechadas com o paciente, o cuidado está conectado a várias dimensões, envolvendo vários atores e outros trabalhos. É interdependente de uma complexa rede de relações, protagonismos, múltiplos agires, ainda que se tenha a ilusão de estar a sós com ele. A equipe de saúde é responsável por um número de pessoas a serem cuidadas, de acompanhá-las, estar sensível as suas necessidades e ir oferecendo as possibilidades de cuidado(25).

O cuidado nas organizações de saúde em geral, em particular nos hospitais, é multidisciplinar ${ }^{(16)}$. Entretanto, mecanismos de dominação e de relações assimétricas de poder entre os trabalhadores ocultam a imprescindível colaboração que deveria existir entre os profissionais, como operadores de tecnologia de saúde, para que o cuidado se efetive.

Nesse sentido, é um desafio aos profissionais da equipe de saúde e, em especial ao médico, o repensar do papel de responsabilidade individual avançando para a corresponsabilização frente o cuidado das pessoas. Em determinadas circunstâncias, tem-se que aprender a recomeçar. Esse processo perpassa pela mudança de atitude, pelo cuidado responsável, sensível e ético, que tem como propósito a valorização da vida do ser humano que está sendo cuidado. Ao humanizar o seu olhar de profissional, pode estar reeducando a sua sensibilidade, flexibilidade, aprofundar o diálogo e a criação de vínculos nas relações e no processo do cuidado hospitalar(24). 
A construção de um bom diálogo é um passo fundamental e estratégico para sistematizar as ações de processo-doença. Familiares e amigos também são peças fundamentais, pois o paciente sente-se mais protegido. Para estimular a participação dos familiares no tratamento do paciente é preciso construir um vínculo não somente com o paciente, mas com os familiares, devendo criar diálogo proporcionando segurança e conforto a família(11).

\section{CONCLUSÃO}

O estudo aponta que grande parte dos entrevistados é formada por pacientes idosos, que não possuem o ensino fundamental completo, cujas condições podem dificultar a construção do conhecimento a respeito do diagnóstico que resultou na internação bem como a finalidade dos cuidados necessários para tanto; confirmando uma das hipóteses apontadas.

Identifica-se que os pacientes internados conhecem o (s) diagnóstico (s) que resultou (ram) na internação e o plano terapêutico, e que essas orientações foram transmitidas pelo médico. No entanto, esta ação se transcorreu de forma mecanizada, ou seja, não foi possível identificar relação dialógica entre o profissional de saúde e usuário do serviço. Assim, evidenciou-se a dificuldade dos trabalhadores em operacionalizar sua prática na direção da integralidade em saúde e do trabalho em equipe.

Evidenciou-se que existem diversas maneiras para criar/construir o vínculo profissional de saúde-paciente, e uma das principais formas é a comunicação efetiva.

Diante deste cenário, acredita-se que se faz necessário qualificar os profissionais na perspectiva da integralidade do cuidado em saúde e do trabalho em equipe, para tanto, há de se instituir a Política de Educação Permanente em Saúde. Conclui-se também que novos estudos devem ser realizados no sentido de contribuir para a efetivação da autonomia dos pacientes durante a sua hospitalização.

\section{REFERÊNCIAS}

1. Pereira MM, Rezende KTA, Santos IF, Tonhon SFR. Compreensão do Paciente Sobre o seu Processo de Hospitalização. Atas CIAIQ 2018 Investigação Qualitativa em Saúde [Internet]. 2018;2:10. Disponível em: https://proceedings.ciaiq.org/index.php/ciaiq2018/article/view/1853/1803

2. Galvan DC, Kaufmann G, Brustolin AM, Ascari RA. Percepção dos pacientes acometidos pela leucemia frente à internação hospitalar. Rev Enferm da UFSM [Internet]. 2013;3:647-57. [Acesso em 2016 Abr 30]. Disponível em: https://periodicos.ufsm.br/reufsm/article/view/11079/pdf

3. Maniva SJCF, Freitas CHA, Jorge MSB, Carvalho ZMF, Moreira TMM. Vivendo o acidente vascular encefálico agudo: significados da doença para pessoas hospitalizadas. Rev Esc Enferm da USP [Internet]. 2013;47(2):362-8. [Acesso em 2016 Abr 30]. Disponível em: http://www.scielo.br/pdf/reeusp/v47n2/13.pdf

4. Parcianello MK, Fonseca GGP, Zamberlan C. Necessidades vivenciadas pelos pacientes pós-cirurgia cardíaca: percepções da enfermagem. Rev de Enferm Cent-Oes Min [Internt]. 2011;(3):305-12. [Acesso em Abr 30]. Disponível em http://seer.ufsj.edu.br/index.php/recom/article/view/89

5. Santos HGS, Santos CIL, Lopes DFM, Belei RA. Multirresistência bacteriana: a vivência de pacientes internados em hospital escola do município de Londrina - PR. Cienc, Cuid e Saud [Internet]. 2010;9(1):74-80. [Acesso em Abr 30]. Disponível em: http://periodicos.uem.br/ojs/index.php/CiencCuidSaude/article/view/7178

6. Proença MO, Dell Agnolo CM. Internação em Unidade de Terapia Intensiva: percepção de pacientes. Rev Gaúc de Enferm [Internet]. 2011;32(2):279-86. [Acesso em Mai 1]. Disponível em: http://www.scielo.br/scielo. php?script=sci_arttext\&pid=S1983-14472011000200010

7. Minayo MCS. O desafio do conhecimento: pesquisa qualitativa em saúde. São Paulo: Hucitec; 2013.

8. HCFAMEMA [homepage na internet]. Hospital das Clínicas - HC I. [Acesso em 2016 Mai 15]. Disponível em: http://www.famema.br/assistencial/index.php.

9. Campos GWS, Amaral MA. A clínica ampliada e compartilhada, a gestão democrática e redes de atenção como referenciais teórico-operacionais para a reforma do hospital. Ciênc e Saúd Col [Internet]. 2007;12(4):849-59. [Acesso em 2016 Mai 15]. Disponível em: http://www.scielo.br/scielo.php?pid=S14138123 $2007000400007 \&$ script=sci_abstract\&tlng $=p t$ 
10. Hafner MLMB, Moraes MAA, Marvulo MML. Braccialli LAD, Carvalho MHR, Gomes R. A formação médica e a clínica ampliada: resultados de uma experiência brasileira. Rev Ciênc e Saúd Colet [Internet]. 2010;15(1):1715-24. [Acesso em 2016 Mai 16]. Disponível em: http://www.scielo.br/scielo.php?pid=S1413812 32010000700083\&script=sci_abstract\&tlng=pt

11. Branco LASC, Maia NMFS, Lima LAA. A construção do vínculo enfermeiro-cliente pelo diálogo no ambiente hospitalar. Rev Enferm UFPI [Internet]. 2016;5(3):30-5. [Acesso em 2016 Mai 16]. Disponível em: http://www. ojs.ufpi.br/index.php/reufpi/article/view/5436/pdf

12. Brandão ALRBS, Giovanella L, Campos CEA. Avaliação da atenção básica pela perspectiva dos usuários: adaptação do instrumento EUROPEP para grandes centros urbanos brasileiros. Ciênc Saúde Colet [Internet]. 2013;18(1):103-14. [Acesso em 2016 Mai 31]. Disponível em: http://www.scielo.br/pdf/csc/v18n1/12.pdf

13. Membrive AS, Souza LPS, Donoso MTV, Silqueira SMF, Corrêa AR, Matos SS. Caracterização dos estressores envolvidos na internação de pacientes em unidade coronariana. Rev Baiana Enferm [Internet]. 2017;31(1):16552. [Acesso em 2016 Mai 31]. Disponível em: https://portalseer.ufba.br/index.php/ enfermagem/article/view/16552/14150

14. Dubow C, Olivo VMF, Ceron MI, Vedootto DO, Dal Moro JS, Oliveira CP, Barbieri A. Linha de cuidado como dispositivo para a integralidade da atenção a usuários acometidos por agravos neoplásicos de cabeça e pescoço. Saúde e Debate [Internet]. 2014;38(100):94-103. [Acesso em 2016 Mai 16]. Disponível em: http:// www.scielo.br/scielo.php?pid=S0103-11042017000401177\&script=sci_abstract

15. Fernandes MJC, Silva AL. A pessoa idosa em estado crítico: barreiras ao cuidado integral. Rev enferm UFPE [Internet]. 2018;12(7):1887-99. [Acesso em 2016 Mai 31]. Disponível em: https://periodicos.ufpe.br/revistas/ revistaenfermagem/article/view/235007/29466

16. Cecilio LCO, Merhy EE. A integralidade do cuidado como eixo da gestão hospitalar. In: Construção da integralidade: cotidiano, saberes e práticas em saúde. Rio de Janeiro: IMS ABRASCO; 2003.

17. Biasibetti C, Hoffmann LM, Rodrigues FA, Wegner W, Rocha PK. Comunicação para a segurança do paciente em internações pediátricas. Rev Gaúcha Enferm [Internet]. 2019;40:1-9. [Acesso em 2016 Mai 30]. Disponível em: http://www.scielo.br/pdf/rgenf/v40nspe/en_1983-1447-rgenf-40-spe-e20180337.pdf

18. Brasil. Ministério da Saúde. Secretaria de Atenção à Saúde. Política Nacional de Humanização da Atenção e Gestão do SUS. Clínica ampliada e compartilhada [Internet]. Brasília, 2009. [Acesso em 2016 Mai 27]. Disponível em: http://bvsms.saude.gov.br/bvs/publicacoes/clinica_ampliada_compartilhada.pdf

19. Clotet J. O respeito à autonomia e aos direitos dos pacientes. Revista da AMRIGS [Internet]. 2009;53(4):43235. [Acesso em 2016 Mai 27]. Disponível em: http://www.amrigs.org.br/revista/53-04/22-534-respeito \%C3\%A0-autonomia.pdf

20. Calegari RC, Massarollo MCKB, Santos MJ. Humanização da assistência à saúde na percepção de enfermeiros e médicos de um hospital privado. Rev Escola de Enferm da USP [Internet]. 2015;49(2):42-7. [Acesso em 2016 Mai 28]. Disponível em: http://www.scielo.br/pdf/reeusp/v49nspe2/1980-220X-reeusp-49spe2-0042.pdf

21. Carretta MB, Bettinelli LA, Erdmann AL. Reflexões sobre o cuidado de enfermagem e a autonomia do ser humano na condição de idoso hospitalizado. Rev Bras de Enferm [Internet]. 2011;64(5). [Acesso em 2016 Mai 26]. Disponível em: http://dx.doi.org/10.1590/S0034-71672011000500024

22. Pott FS, Stahlhoefer T, Felix JVC, Meier MJ. Medidas de conforto e comunicação nas ações de cuidado de enfermagem ao paciente crítico. Rev Bras de Enferm [Internet]. 2013;66(2):174-79. [Acesso em 2016 Mai 25]. Disponível em: http://www.scielo.br/scielo.php?script=sci_abstract\&pid=\$0034-71672013000200004\&lng =en\&nrm=iso>

23. Siqueira PG, Silva RM da, Beck CLC, Prestes FC, Vedootto DO, Pasa TS. Percepção de usuários hospitalizados sobre o atendimento nos serviços de saúde. Rev Enferm UFSM [Internet]. 2016;6(4):471-81. [Acesso em 2019 Jun 01]. Disponível em: http://dx.doi.org/10.5902/2179769222355

24. Bettinelli LA, Carretta MB, Marcolin D, Poleto S, Brock F, Antonioli NS. Autonomia e a dependência: Experiências de Idosos na Hospitalização. Rev Contexto e Saúde [Internet]. 2011;10(20):727-32. [Acesso em 2016 Mai 26]. Disponível em: https://www.revistas.unijui.edu.br/index.php/contextoesaude/article/view/1638 
25. Cecilio L. (2015). Curso de aperfeiçoamento em Saúde Mental - módulo 4 - o cuidado em saúde. UNA-SUS/ UNIFESP: 2015.

\section{Endereço do primeiro autor:}

Marcella Maria Pereira

Faculdade de Medicina de Marília

Av. Monte Carmelo, 800

Bairro: Fragata

CEP: 17519-030 - Marília - SP - Brasil

E-mail: marcellamariap@gmail.com

Endereço para correspondência:

Katia Terezinha Alves Rezende

Faculdade de Medicina de Marília

Av. Monte Carmelo, 800

Bairro: Fragata

CEP: 17519-030 - Marília - SP - Brasil

E-mail: katia@famema.br 